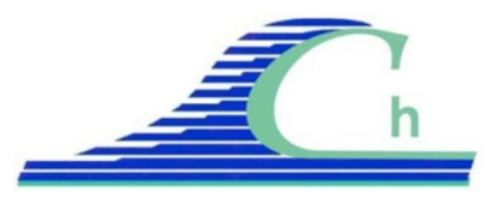

XII ${ }^{\text {ìmes }}$ Journées Nationales Génie Côtier - Génie Civil

Cherbourg, 12-14 juin 2012

DOI:10.5150/jngcgc.2012.027-D @ Editions Paralia CFL

disponible en ligne - http://www.paralia.fr - available online

\title{
Modélisation de l'évolution des profils de plage sableuse sur plusieurs mois et apports de l'assimilation de données
}

\author{
Benjamin DUBARBIER ${ }^{1}$, Bruno CASTELLE ${ }^{1}$, Florent BIRRIEN ${ }^{1}$, \\ Vincent MARIEU ${ }^{1}$, Gerben RUESSINK ${ }^{2}$
}

1. CNRS UMR EPOC-5805, Université de Bordeaux 1, Avenue des Facultés, 33405 Talence Cedex, France.

b.dubarbier@epoc.u-bordeaux1.fr ; b.castelle@epoc.u-bordeaux1.fr

v.marieu@epoc.u-bordeaux1.fr ; f.birrien@epoc.u-bordeaux1.fr

2. Institute for Marine and Atmospheric Research, Department of Physical Geography, Faculty of Geosciences, Utrecht University, The Netherlands.

B.G.Ruessink@uu.nl

\section{Résumé :}

Dans ce papier nous présentons un nouveau modèle de profil de plage sableuse réalisant le couplage vagues/transport sédimentaire/évolution bathymétrique avec une approche à phase moyennée. Nous utilisons une paramétrisation récente des effets non-linéaires des vitesses orbitales des vagues au fond permettant d'améliorer les flux sédimentaires vers la côte. Nous validons le modèle numérique pour un cas de migration de barres sableuses vers le bord (Duck, Caroline du Nord) et vers le large (Egmond, Pays-Bas) sur une période de plusieurs jours. Nous montrons que le modèle reproduit avec fidélité l'évolution des profils mesurés en termes de migration et de morphologie, confirmée par des indices de performance (Brier skill) supérieures à 0.5 en fin de chaque simulation. Certaines limitations comme les erreurs en domaine intertidal sont discutées ainsi qu'un cas synthétique d'apport de l'assimilation de données.

Mots-clés : Barres sableuses - Migration - Modèle à phase moyennée - Asymétrie des vagues - Transport sédimentaire

\section{Abstract:}

This paper presents the development of a simple coupled, wave-averaged, cross-shore waves-currents-bathymetry evolution model. A recent parameterization of the freestream nonlinear wave orbital motion is used to improve onshore sediment transport. We compare the model with data gathered at two natural beaches where sandbars move onshore (Duck) and offshore (Egmond) on the timescales of weeks. Reliable simulations of observed sandbar behavior (migration and shape) are obtained, despite mismatch at the upper part of the beach. Synthetic assimilation test cases are performed to overcome missing swash-processes, and are further discussed.

Keywords : Sandbar - Process-based model - Velocity skewness - Wave-driven sediment transport 


\section{Introduction}

La compréhension de la dynamique des profils de plages sableuses est essentielle pour prévoir l'érosion durant les épisodes de tempête et l'impact des projets de construction côtière, de dragages ou de rechargement. L'évolution morphologique du fond sableux est le résultat de l'interaction de nombreux processus hydro-sédimentaires qui interagissent dans la zone littorale à différentes échelles spatio-temporelles. Les barres sableuses, fréquemment observées le long des littoraux sableux, sont des structures sédimentaires complexes qui représentent une protection naturelle des plages en dissipant l'énergie des houles par déferlement bathymétrique. GALLAGHER et al. (1998) ont montré que la migration rapide des barres sableuses vers le large lors d'épisodes énergétiques était principalement contrôlée par le courant de retour induisant un transport sédimentaire important vers le large. Au contraire, HOEFEL \& ELGAR (2003) ont mis en évidence que, pendant les épisodes de temps calme, les asymétries des vagues induisent un flux sédimentaire net vers la côte contrôlant la migration lente des barres sableuses vers la plage.

Les modèles numériques basés sur une approche à phase moyennée, réalisant le couplage entre l'hydrodynamique (vagues et courant), le transport sédimentaire et l'évolution bathymétrique sont récemment parvenus à reproduire de façon acceptable l'évolution de profil de plage sur des échelles de temps allant de plusieurs semaines (RUESSINK et al., 2007) à quelques années (WASLTRA et al., 2011). Néanmoins, la précision de ces modèles est limitée par une description insuffisante des processus physiques impliqués à différentes échelles spatio-temporelles. En particulier, la dynamique complexe dans la zone jet de rive n'est actuellement pas prise en compte dans les modèles à phase moyennée induisant de fortes erreurs de l'évolution du haut de plage. C'est pourquoi le développement de ce type de modèle est essentiel tout comme l'utilisation de méthodes d'assimilation de données qui peuvent pallier ces problèmes.

Dans ce papier, nous décrivons le modèle numérique et discutons de la validité des nouvelles paramétrisations mises en place. Nous montrons ensuite des cas d'application du modèle pour des migrations de barres sableuses vers le large et vers le bord sur des échelles de temps de plusieurs mois. Certaines limitations, comme par exemple les erreurs en domaine intertidal, seront discutées ainsi qu'un cas synthétique d'apport de l’assimilation de données.

\section{Description du modèle}

Ce modèle d'évolution de profil de plage sableuse réalise le couplage vagues/transport sédimentaire / évolution bathymétrique. L'approche unidimensionnelle suppose que les iso-contours bathymétriques de la plage sont uniformes et parallèles à la côte. Le modèle se compose de deux modules principaux que nous décrivons ci-dessous. 


\section{XII ${ }^{\text {èmes }}$ Journées Nationales Génie Côtier - Génie Civil \\ Cherbourg, 12-14 juin 2012}

\subsection{Module hydrodynamique}

Le module hydrodynamique (pas détaillé ici) utilise l'équation de conservation du flux d'énergie des vagues en utilisant le modèle M2 de THORNTON \& GUZA (1983) et le modèle de rouleau de déferlement de DALLY \& BROWN (1995). Les effets de cisaillement de vent en surface et de frottement sur le fond sont négligés.

Du fait de l'importance des courants moyens dans les flux sédimentaires, nous avons utilisé le modèle analytique 1DV introduit par RENIERS et al. (2004) qui permet de reproduire la structure verticale du courant moyen dans la couche limite de fond ainsi que dans la couche d'eau entre la couche limite de fond et le niveau moyen du creux des vagues. Le déséquilibre entre les gradients horizontaux de pressions et de tensions de radiation induit un écoulement des masses d'eau vers le large (courant de retour) qui compense le flux de masse des vagues (mouvement organisé et rouleau de déferlement). Nous utilisons le courant moyen au sommet de la couche limite pour calculer dans la suite les flux sédimentaires perpendiculaires à la côte.

\subsection{Module sédimentaire.}

Nous utilisons l'approche énergétique développée par HSU et al. (2006) qui permet de dissocier le transport sédimentaire induit par les effets non-linéaires des vitesses orbitales au fond (asymétrie horizontale (skewness) et verticale), du transport induit par le courant moyen. Par ailleurs, nous n’incluons pas ici les flux sédimentaires générés par l'accélération du fluide au fond (HOEFEL \& ELGAR, 2003) générant des flux de sédiments supplémentaires vers la côte :

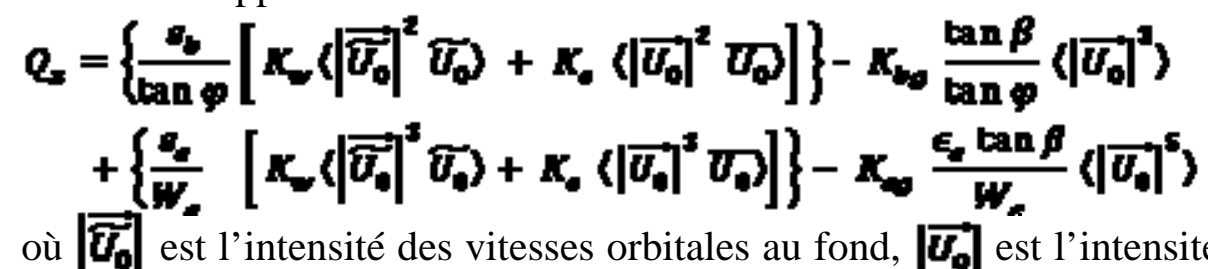
(courant moyen et vitesse orbitale), $\beta$ l'angle local de la pente de plage, $\varphi$ l'angle de friction interne des sédiments, $W_{s}$ leur vitesse de chute, les coefficients $\varepsilon_{b}$ et $\varepsilon_{s}$ sont des facteurs d'efficacité du transport sédimentaire par charriage et par suspension, $K_{W}$ et $K_{c}$ sont respectivement les coefficients de frottement relatif au transport induit par les vitesses orbitales et le courant moyen et sont considérés comme deux paramètres ajustables dans ce modèle, enfin $K_{b g}$ et sont $K_{s g}$ des facteurs de frottement liés au transport par effet de pente. Le modèle de vague à phase moyennée ne permet pas d'obtenir explicitement les termes $\left|\overrightarrow{\boldsymbol{v}_{0}}\right|$ et $\left|\overrightarrow{\boldsymbol{U}_{\boldsymbol{\phi}}}\right|$, par conséquent nous utilisons la paramétrisation récente proposée par RUESSINK et al (soumis), qui propose la calibration de deux facteurs adimensionnels qui caractérisent les effets non-linéaires des vagues en zone de levée et de déferlement en fonction du nombre d’Ursell.

Cette approche utilise la solution analytique proposée par ABREU et al. (2010) pour reconstruire la série temporelle des vitesses orbitales en chaque point du maillage : 


$$
D_{0}(t)=U_{v} f \frac{\left[\sin (\omega t)+\frac{r \sin \phi}{1+\sqrt{1-r^{2}}}\right]}{[1-r \cos (w t+\phi)]}
$$

où $r$ est une mesure des non-linéarités, $f$ un paramètre adimensionnel limitant l'amplitude de la vitesse orbitale, $\phi$ est la phase, $\omega$ la pulsation et $U_{W}$ l'amplitude des vitesses orbitales au fond déterminée par la théorie linéaire. L’objectif de cette paramétrisation est de calibrer les coefficients $r$ et $\phi$ afin d'obtenir des asymétries horizontales et verticales des séries temporelles monochromatiques $\boldsymbol{\nabla}_{\mathbf{N}}(\mathbf{r})$ représentatives d'un champ de vagues aléatoires en milieu naturel. Le résultat de cette calibration est montrée figure 1. Dans cet exemple, nous voyons que plus la valeur locale du nombre d'Ursell est importante, plus les effets non-linéaires sont dominants. Ainsi, au large à la position 1 la série temporelle des vitesses orbitales est purement sinusoïdale. Au-dessus de la barre externe à la position 2, la vitesse devient à la fois asymétrique horizontalement et verticalement. La valeur du nombre d'Ursell étant plus faible dans la fosse, la vitesse orbitale redevient strictement sinusoïdale et enfin, en zone de surf interne (position 4), les effets non-linéaires importants induisent des vitesses quasiment en dent de scie, signature des fronts d'ondes déferlées.

L'élévation du fond sableux est calculée à partir de la résolution de l'équation de conservation du sédiment avec un schéma numérique de type NOCS sur maillage décalé.
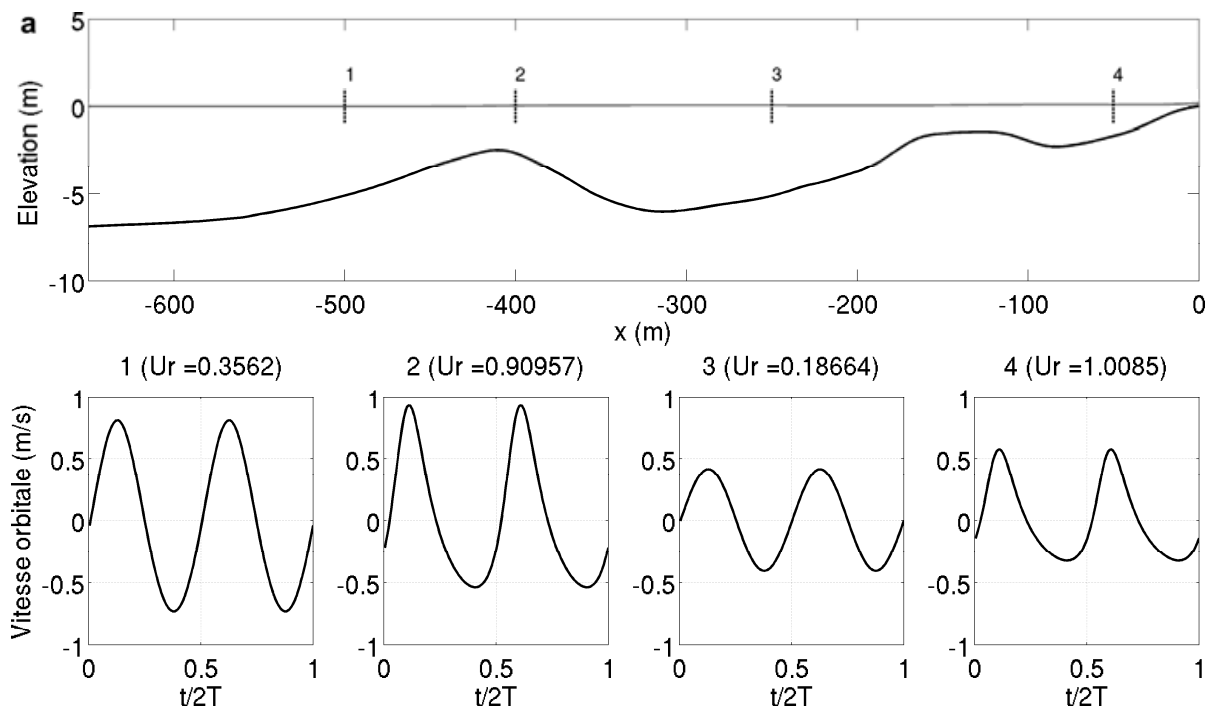

Figure 1. a) Profil de plage d'Egmond (Pays-Bas), la ligne grise représente l'élévation moyenne de la surface libre pour des conditions de houle au large correspondant à

$H r m s=1.5 m, T p=8 \mathrm{~s}$. Les figures numérotées de 1 à 4 représentent les séries temporelles de vitesse orbitale au fond aux positions correspondantes indiquées audessus. 


\section{XII ${ }^{\text {èmes }}$ Journées Nationales Génie Côtier - Génie Civil \\ Cherbourg, 12-14 juin 2012}

2.2 Module d'assimilation.

Les méthodes d'assimilation peuvent être utilisées pour corriger des modèles de prédictions d'évolution de plage dans le cas de suivi long terme d'une zone littorale. Elles utilisent soit des observations directes acquises in situ, i.e. relevés bathymétriques/topographiques ou dérivées d'imagerie vidéo, avec lesquelles il est possible de reconstruire une cartographie du fond à partir de méthodes d'inversion. Le module d'assimilation est implémenté en parallèle du modèle d'évolution de profil de plage, il utilise un schéma d'assimilation basé sur un filtre de Kalman. Des observations (directes ou indirectes) permettent de corriger les données du modèle afin de reconstruire un nouveau profil (BIRRIEN et al., 2011).

\section{Résultats}

Le modèle présenté est confronté à des relevés bathymétriques effectués durant deux campagnes de mesures, l'une sur la plage de Duck (Caroline du Nord, 1982) et la deuxième sur la plage d'Egmond (Pays-Bas, 1998). La calibration de chaque simulation est effectuée en trouvant une combinaison de 3 paramètres ajustables $\left(K_{W}, K_{C}\right.$ et un facteur adimensionnel dans le modèle de dissipation M2) qui minimisent au mieux l'erreur quadratique entre les observations et les résultats du modèle en respectant la gamme de validité de chaque paramètre (GALLAGHER et al., 1998). Pour évaluer la validité des simulations nous utilisons un indice de performance (MPI) de type Brier skill (RUESSINK et al., 2007).

3.1 Episode de migration d'une barre sableuse vers la côte sur plusieurs semaines (Duck, C.N)

Durant la campagne de mesures Duck1982, le système simple barre a migré de $65 \mathrm{~m}$ vers la côte sur une période de 3.5 mois. Durant cette migration, l’amplitude de la barre a diminué de moitié réduisant ainsi le relief entre la barre et la fosse. Les conditions de houle au large sont données à une profondeur de $-8 \mathrm{~m}$, et la simulation effectuée couvre la totalité de cette période avec un pas de temps entre chaque itération morphodynamique fixé à 30 minutes.

Le modèle reproduit avec fidélité l'évolution de profil en termes de migration et de morphologie dans la zone où la barre est active $(-400<\mathrm{x}<-100 \mathrm{~m})$. Ceci est confirmé par un indice de performance proche de 1 en fin de simulation (1 étant la valeur indiquant un modèle parfait). Nous reproduisons également la diminution de moitié de l'amplitude de la barre dans le temps. Néanmoins, le modèle peine à reproduire l'évolution du haut de plage ce qui peut s'expliquer en partie par le fait que les processus impliqués dans la zone de jet de rive ne sont pas pris en compte. 

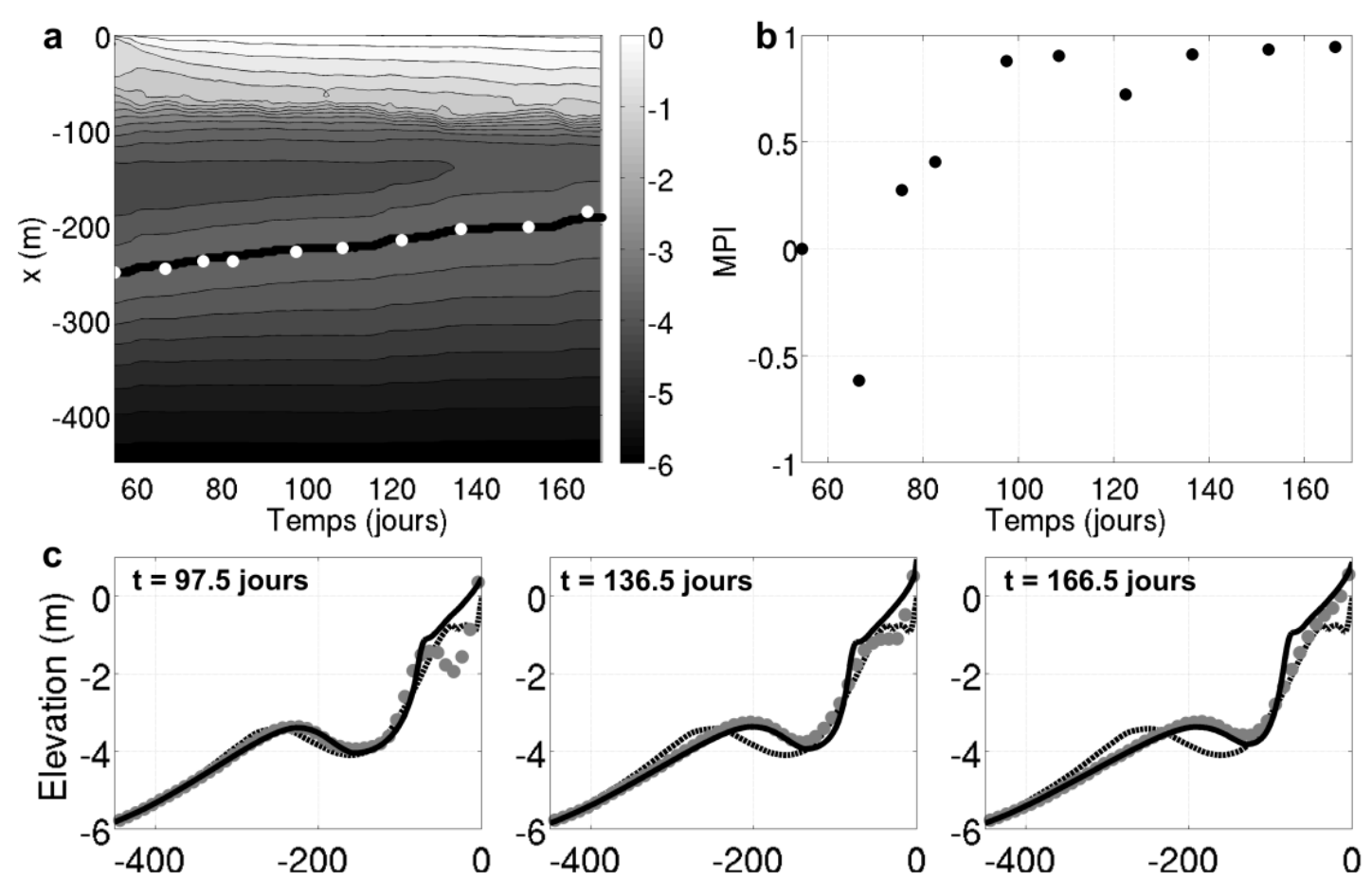

Figure 2. a) Prédiction de l'évolution spatio-temporelle du profil de plage de Duck1982, les points blancs indiquent les positions de la crète de la barre mesurées et les points noirs celles simulées. b) Indice de performance du model MPI et c) profil de plage simulé en trait plein noir et mesuré en point gris à différent temps, le profil initial est en trait pointillé noir.

3.2 Episode de migration d'une barre sableuse vers le large sur plusieurs jours (Egmond, NL)

La campagne de mesures sur le site d'Egmond (Pays-Bas) a permis d'étudier un système double barres dont la barre interne présente des irrégularités quasi-périodiques le long de la côte tandis que le barre subtidale est uniforme sur plusieurs kilomètres le long de la côte. Nous disposons d'un set de données qui s'étend sur une durée de 34 jours, comprenant la série temporelle du forçage de vagues à une profondeur de -18m et de 6 relevés bathymétriques s’étendant jusqu’à 600m au large.

Les résultats de la simulation montrent que la migration vers le large de la barre externe est correctement reproduite (figure 3a,c), bien que nous sous-estimions son amplitude d'environ $40 \mathrm{~cm}$ en fin de simulation. Néanmoins, l'indice de performance calculé entre -450 et $-300 \mathrm{~m}$, correspondant à la zone active de la barre externe, donne un résultat supérieur durant la quasi-totalité de la simulation (figure 3b, ronds vides) comparé aux deux autres MPI effectués respectivement entre -200 et -30 m couvrant uniquement la zone de la barre interne (figure $3 \mathrm{~b}$, triangles) et -450 à $-30 \mathrm{~m}$ englobant les deux barres 


\section{XII ${ }^{\text {èmes }}$ Journées Nationales Génie Côtier - Génie Civil \\ Cherbourg, 12-14 juin 2012}

(figure 3b, ronds pleins). Nous soulignons que le MPI de la barre interne conserve une valeur positive et supérieure à 0.5 en fin de simulation.
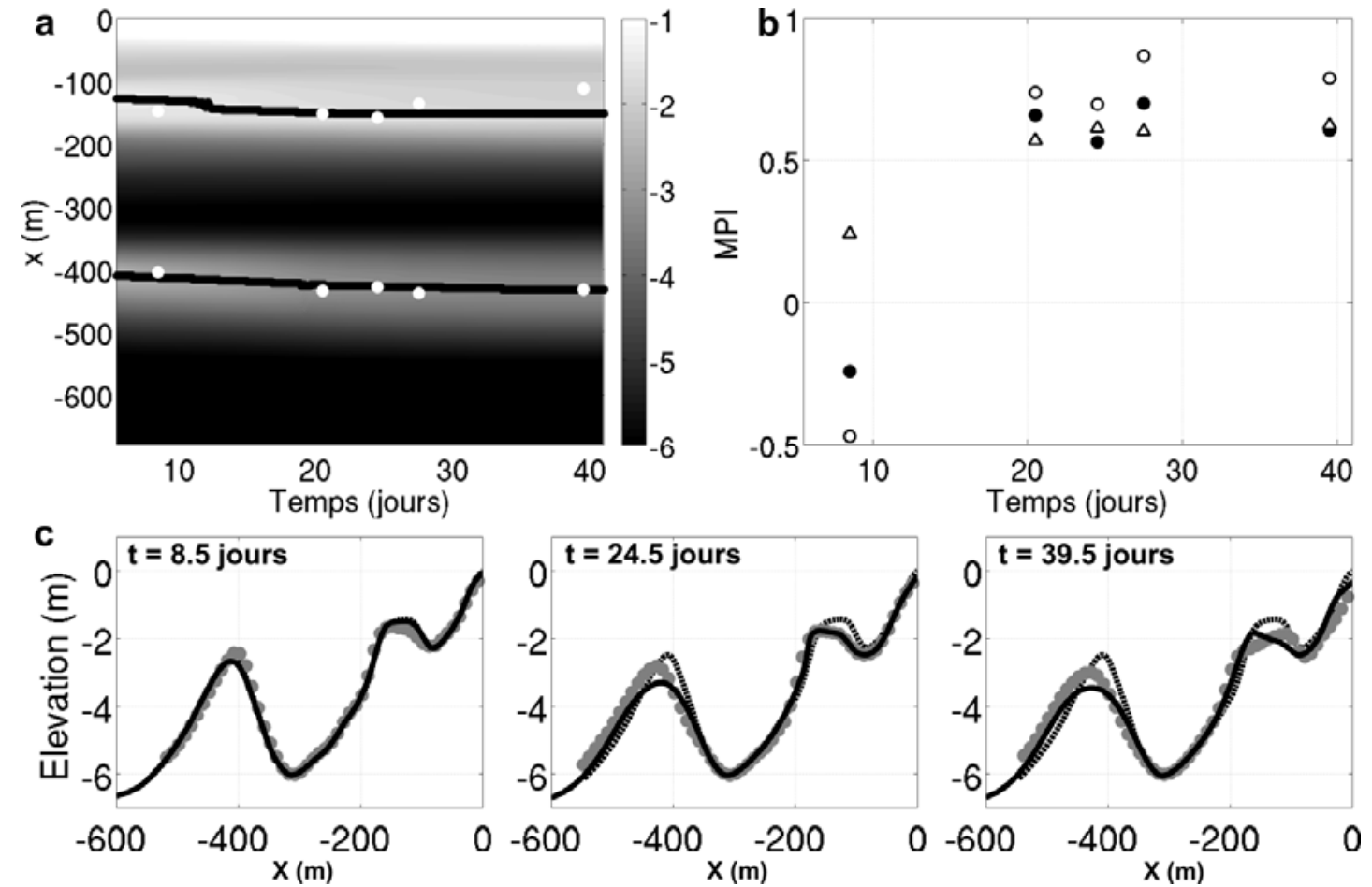

Figure 3. a) Prédiction de l'évolution spatio-temporelle du profil de plage d'Egmond, les points blancs indiquent les positions de la crète de la barre mesurées et les points noirs celles simulées. b) Indice de performance calculé entre -450 à -30 m (ronds noirs), -200 à -30 m (triangles) et -450 à -300 m (rond blancs) et c) profil de plage simulé en trait plein noir et mesuré en point gris à différent temps, le profil initial est en trait pointillé noir.

\subsection{Cas synthétique d’application de l'assimilation de données}

Dans le but d'améliorer la prédiction du modèle notamment en haut de plage, nous utilisons en parallèle un module d'assimilation permettant de corriger une partie du profil de plage avec les relevés bathymétriques servant d'opérateur d'observation dans le schéma d'assimilation. L’idée est de réitérer les simulations précédentes avec les mêmes paramétrisations et d'effectuer une assimilation en utilisant comme données d'observation le profil de plage intertidal (Duck) et la zone couvrant la barre interne et le haut de plage (Egmond).

Nous constatons dans les deux cas d'étude qu'au temps où nous effectuons une assimilation (figure 4a,c) le profil antérieur à la phase d'assimilation (trait pointillés noirs) converge parfaitement vers les observations à la fin de cette étape (trait plein noir) en quelques itérations. En comparant les prédictions du modèle au temps d'observation post-assimilation, environ une dizaine de jours plus tard, nous observons 
(figure 4b) une rapide divergence de l'évolution du haut de plage mais surtout une très faible différence avec la simulation sans assimilation (trait pointillé gris). Nous supposons ici que la pente du haut de plage étant significative, de l'ordre de 1:20, l'impact du backswash doit jouer un rôle important dans la redistribution du sédiment vers le large. En effet l'évolution du haut de plage simulée tend vers une pente à l'équilibre d'environ 1:50, reflétant un transport net de sédiments vers la côte qui induit une accrétion du haut de plage. Dans le cas d'Egmond (figure 4b) l'apport de l'assimilation est au contraire plus efficace car le profil simulé reste proche de la bathymétrie mesurée à la différence de la simulation sans étape d'assimilation qui sousestime la profondeur de la fosse interne.
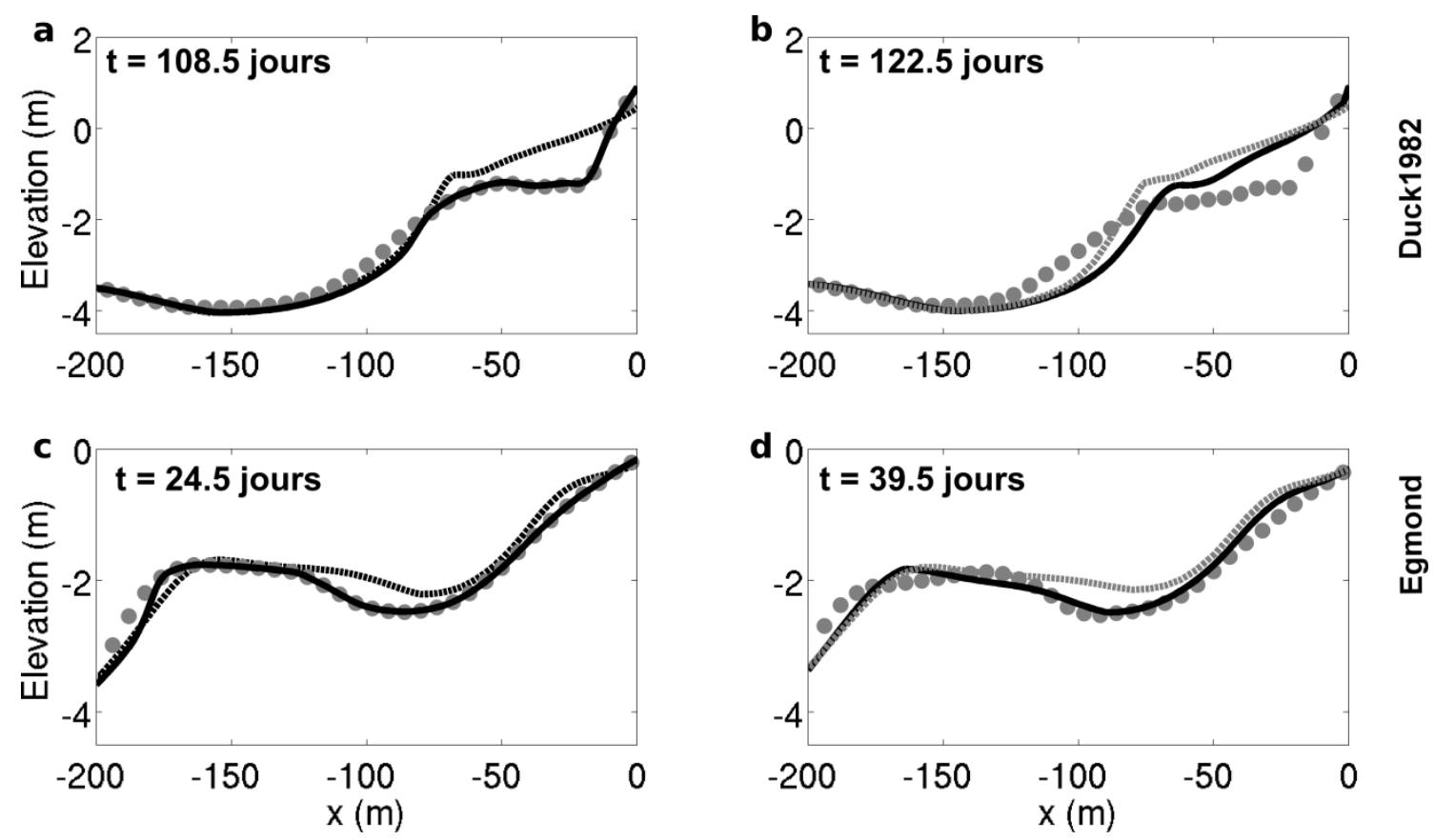

Figure 4.a-c) Profil de plage avant étape d'assimilation (trait pointillé noir) comparé au profil post-assimilation (trait plein noir) et bathymétrie d'observation (ronds gris) .b-d) Profil de plage simulé avec étape d'assimilation (trait noir) comparé au profil de plage simulé sans étape d'assimilation (trait pointillé gris) et bathymétrie d'observation (ronds gris).

\section{Conclusion}

Ce papier présente un modèle d'évolution de profil de plage basé sur le couplage entre un modèle de vague à phase moyennée et une paramétrisation simple des flux sédimentaires, permettant d'effectuer des simulations morphodynamiques sur des périodes de temps allant de plusieurs jours à quelques mois avec des temps de calculs très courts. Une calibration différente de trois paramètres ajustables nous permet dans un premier temps de simuler sur le site de Duck un cas d'épisode de migration de barre 


\section{XII ${ }^{\text {èmes }}$ Journées Nationales Génie Côtier - Génie Civil \\ Cherbourg, 12-14 juin 2012}

sableuse vers la côte de manière très satisfaisante, ceci étant confirmé par un indice de performance du modèle proche de 1 en fin de simulation. Dans un deuxième temps, nous simulons un cas de migration de barre vers le large sur le site d'Egmond dans lequel nous observons une vitesse de migration de la barre externe correctement reproduite malgré une sous-estimation de son amplitude de $40 \mathrm{~cm}$ en fin de simulation. Dans les deux cas d'études, la prédiction de l'évolution du haut de plage reste problématique étant donné que l'approche énergétique utilisée pour le calcul des flux sédimentaires n'est pas adaptée aux processus instationnaires de la zone de jet de rive. Pour réajuster la précision du modèle en haut de plage nous utilisons une méthode d'assimilation de données bathymétriques efficace mais qui ne permet pas dans le temps de pallier le manque de processus physique intervenant dans la zone intertidale. A terme des expériences en canal à houle vont être réalisées pour permettre d'améliorer la modélisation des processus hydro-sédimentaires impliqués dans la zone de surf.

\section{Remerciements}

Ces travaux sont réalisés dans le cadre du projet ANR BARBEC. Nous remercions l'U.S. Army Corps of Engineers Field Research Facility pour avoir collecté les données sur la plage de Duck et de les avoir rendues publiques.

\section{Références bibliographiques}

ABREU T., SILVA P., SANCHO F., TEMPERVILLE A. (2010). Analytical approximate wave form for asymmetric waves. Coastal Engineering, 57, pp 656-667. doi:10.1016/j.coastaleng.2010.02.005

BIRRIEN F., CASTELLE B., MARIEU V., ALMAR R., MICHALLET H. (2011). Application of a data-model assimilation method to a $3 D$ surf zone sandbar physical experiment. Journal of Coastal Research, pp 976-980.

DALLY W., BROWN C.A. (1995). A modeling investigation of the breaking wave roller with application to cross-shore currents. Journal of Geophysical Research, 100, pp 24873-24883. doi:10.1029/95JC02868

GALLAGHER E.L., ELGAR S., GUZA R.T. (1998). Observations of sand bar evolution on a natural beach. Journal of Geophysical Research, 103, pp 3203-3215. doi:10.1029/97JC02765

HOEFEL F., ELGAR S. (2003). Wave-induced sediment transport and sandbar migration. Science, 299, pp 1885-1887. doi:10.1126/science.1081448

HSU T.J., ELGAR S., GUZA R.T. (2006). Wave-induced sediment transport and onshore sandbar migration, Coastal Eng., 53, pp 817-824. doi: 10.1016/j.coastaleng.2006.04.003 RENIERS A.J.H.M., THORNTON E.B., STANTON T.P., ROELVINK J.A. (2004). Vertical flow structure during Sandy Duck: observations and modeling. Coastal Eng., 51, pp 237-260. doi:10.1016/j.coastaleng.2004.02.001 
Thème 2 - Dynamique sédimentaire

RUESSINK B.G., KURIYAMA Y., RENIERS A.J.H.M., ROELVINK J.A., WALSTRA D.J.R. (2007). Modeling cross-shore sandbar behavior on the timescale of weeks. Journal of Geophysical Research, 112, F03010. doi:10.1029/2006JF000730 RUESSINK B.G., RAMAEKERS G, VAN RIJN, L.C (soumis). On the parametrization of the free-stream nonlinear wave orbital motion in coastal morphodynamic models.

THORNTON E.B., GUZA R.T. (1983). Transformation of wave height distribution. Journal of Geophysical Research, 88, pp 5925-5938. doi:10.1029/JC088iC10p05925 WALSTRA D.J.R., RUESSINK B.G., RANASINGHE R. (2011). The influence of initialization on inter-annual model predictions, In Proc. Coastal Sediments'11, pp 859-872. doi:10.1142/9789814355537_0065 